\title{
MESOSPHERIC ELECTRIC FIELD MEASUREMENTS WITH A PARTIAL REFLECTION RADAR
}

\author{
S. I. Martynenko, V. T. Rozumenko, A. M. Tsymbal, O. F. Tyrnov, and A. M. Gokov \\ Department of Space Radio Physics, Kharkiv State University, 4 Svoboda Square, Kharkiv \\ 310077, Ukraine
}

\begin{abstract}
The distribution of variations in the effective electron collision frequency is obtained over the $60-66 \mathrm{~km}$ altitude range in the lower ionosphere (random measurement errors within this altitude range are less than $50 \%$ ). A technique is developed for estimating the variations in atmospheric electric fields at the lower boundary of the ionosphere using the experimental values of the effective electron collision frequency. It follows from our measurements that electric fields of $E>0.25 \mathrm{~V} / \mathrm{m}$ in approximately $70 \%$ of the cases during quiet ionospheric and atmospheric conditions. These results must be taken into account in the investigations of ionospheric processes, meteorological and propagation effects.
\end{abstract}

Key words: intense atmospheric electric fields, mesosphere, partial reflections

\section{Introduction}

Electric fields are fundamental to the dynamics and thermal balance of the atmosphere, ionosphere, and protonosphere. For instance, intense electric fields can produce large disturbances in plasma parameters of the mesosphere (Gurevich, 1978). At present, most of our understanding of the variability of the intense electric fields in the mesosphere come primarily from measurements made by several sounding rockets flown in the last few decades (Goldberg, 1984). At the same time, radar measurements provide considerable advantages in terms of improvements in accuracy and time resolution and enhanced scientific return. Therefore, it will be of central importance to develop a reliable monitoring method to measure the intense electric fields in the mesosphere (Martynenko, 1998).

Our experimental results have revealed that a possible cause of large enough variations in the electron collision frequency could be the effect of external electric fields of atmospheric origin. This provides an opportunity to measure electric fields in the lower ionosphere using remote sensing instruments employing radio-wave techniques.

\section{A technique to derive the effective electron collision frequency and the results}

During 1978-1998 at Kharkiv State University, variations in the effective electron collision frequency, $v$, in the ionospheric D-region have been investigated by means of the partial reflection technique under different solar and geophysical conditions (Tyrnov et al., 1994). The specifications for the radar have been as follows: operating frequency $f=1.8-3.0 \mathrm{MHz}$, pulse length $\tau=25 \mu \mathrm{s}$, pulse repetition rate $F=1$ per second. The data collected on geomagnetically quiet days were chosen for analysis when signal-to-noise ratios were more than 5. Thus, effective electron collision frequency data were accumulated for the altitudes of 60,63 , and $66 \mathrm{~km}$. The transcendental equation in $v$ (see, for example, Martynenko, 1998) 


$$
\frac{\overline{A_{-}^{2}}}{\overline{A_{+}^{2}}}=\frac{\left[\left(\omega+\omega_{L}\right)^{2}+v^{2}\right]^{2}}{\left[\left(\omega-\omega_{L}\right)^{2}+v^{2}\right]^{2}} \cdot \frac{\left(\omega-\omega_{L}\right)^{2} K_{\varepsilon}^{2}\left(\begin{array}{c}
\omega-\omega_{L} \\
v
\end{array}\right)+v^{2} K_{\sigma}^{2}\left(\begin{array}{c}
\omega-\omega_{L} \\
v
\end{array}\right)}{\left(\omega+\omega_{L}\right)^{2} K_{\varepsilon}^{2}\left(\begin{array}{c}
\omega+\omega_{L} \\
v
\end{array}\right)+v^{2} K_{\sigma}^{2}\left(\frac{\omega+\omega_{L}}{v}\right)}
$$

is being solved, where $\overline{A_{-}^{2}}$ is the intensity of the extraordinary mode of partially reflected signals averaged over an 8 to $10 \mathrm{~min}$ interval, and $\overline{A_{+}^{2}}$ is the intensity of the ordinary mode of partially reflected signals averaged over the same interval, $\omega_{L}=2 \pi f_{L}, f_{L}$ is the component of the electron gyrofrequency along the ambient magnetic field direction; in middle latitude experiments, the value of $f_{L}$ is assumed to be equal $1.35 \mathrm{MHz}, K_{\varepsilon}(x)$ and $K_{\sigma}(x)$ are the kinetic coefficients which describe the kinetic effects in the permittivity $\varepsilon$ and conductivity $\sigma$ of the lower ionosphere (Gurevich, 1978). The dependences $K_{\varepsilon}(x)$ and $K_{\sigma}(x)$ can be approximated with an error of an order of a few per cent by the relations

$$
\begin{gathered}
K_{\varepsilon}(x)=1+\frac{a_{1}}{b_{1}+x^{2}} ; \quad a_{1}=0.155, \quad b_{1}=0.075, \quad 0 \leq x \leq \infty ; \\
K_{\sigma}(x)=0.89+\frac{a_{2}}{b_{2}+x^{2}} ; \quad a_{2}=0.027, \quad b_{2}=0.052, \quad 0 \leq x \leq 3.5 ; \\
K_{\sigma}(x)=1 ; \quad 4 \leq x<\infty
\end{gathered}
$$

where $x=\left(\omega-\omega_{L}\right) / v$ for the extraordinary mode, and $x=\left(\omega+\omega_{L}\right) / v$ for the ordinary mode. The partial reflection measurement errors in $v(z)$ do not exceed a magnitude of the order of $30-50 \%$ in the altitude range indicated above.

A histogram derived from the analysis of 92 geomagnetically quiet days of data (collected during the 170 experiments) and showing the distribution of $\nu / v_{m}$ is depicted in Figure 1. Here $v_{m}$ is the model value of $v$ for the same altitude from which partial reflection signals are received; $p=n / n$, where $n$ is the number of observations occurring in each $\nu / \nu_{\mathrm{m}}$ bin, and $n_{l}$ is the number of all the observations (in this case $n_{l}=170$ ). Taking into account the kinetic effects, it was made the assumption that $\nu_{\mathrm{m}}(60 \mathrm{~km})=3.75 \cdot 10^{7}$ $\mathrm{sec}^{-1}, \nu_{\mathrm{m}}(63 \mathrm{~km})=3.55 \cdot 10^{7} \mathrm{sec}^{-1}$, and $\nu_{\mathrm{m}}(66 \mathrm{~km})=1.68 \cdot 10^{7} \mathrm{sec}^{-1}$ (Gurevich, 1978).

If we take into account the fact that fluctuations in the number density of neutral particles and in their temperature in the $\mathrm{D}$ region are generally not more than $10-20 \%$ (in reality their most probable magnitudes are significantly smaller), then the sharp maximum in the $v / v_{\mathrm{m}}$ distribution should be expected within a $(1 \pm 0.2) v / v_{\mathrm{m}}$ value interval. However, taking into account random measurement errors, $70 \%$ of $v / \nu_{\mathrm{m}}$ values exceed the above-mentioned threshold (see Figure 1). From our standpoint, a single reasonable explanation of this result could be the supposition that in $70 \%$ of the cases $T_{c}>T_{n}$ in the lower part of the $\mathrm{D}$ region (where $T_{c}$ is the temperature of electrons, and $T_{n}^{n}$ is the temperature of neutral particles). For natural conditions, the $T_{e}$ relaxation time is less 
than $30 \mu \mathrm{s}$ in the above-mentioned region. Therefore, the most probable cause of longterm existence of increased $T_{e}$ values could be strong atmospheric vertical electric fields (Goldberg, 1984). Based on this assumption, magnitudes of electric fields can be estimated from $\nu / \nu_{\mathrm{m}}$ measurements. Naturally, the cases of $\nu \approx \nu_{m}$ are supposed to correspond to the cases when electric fields are equal to zero, and under this condition the model $v_{m}(z)$-profile have been calculated (Gurevich, 1978).

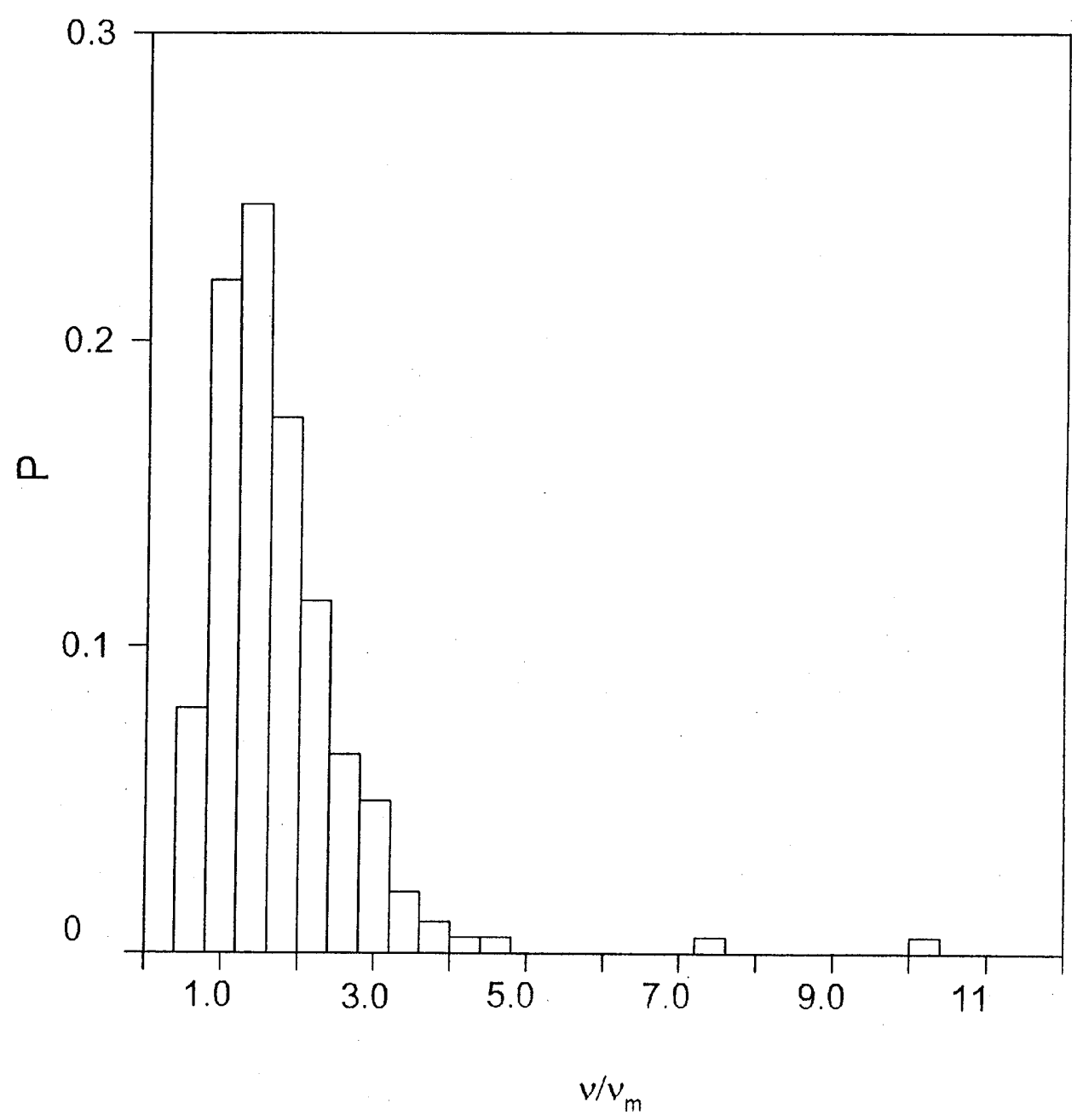

Fig. 1. Distribution of the relative effective electron collision frequency values, $v / v_{m}$, under quiet conditions in an altitude range of $60-66 \mathrm{~km}$ in the daytime lower ionosphere at middle latitudes. 


\section{Technique and electric field measurements}

In order to obtain the dependence of $E$ upon $v$, let us use the results of Martynenko (1998). In a quasi-steady case, $d T_{c} / d t \approx 0$, the relation between electric field intensity, $E$, and $v$ (in Gaussian units) is as follows:

$$
E^{2}=\frac{k n i \delta_{0} T_{e o}}{0.97 e^{2}} \cdot v^{2}\left\{1-\left(\frac{v_{0}}{v}\right)^{6 / 5}\right\}
$$

where

$k$ is Boltzmann's constant, $m$ is the mass of an electron,

$\delta$ is the fractional loss of energy per electron collision,

$e$ is the charge of an electron,

the subscripts " 0 " signify magnitudes of ionospheric parameters in the absence of electric field.

Putting $\delta_{0} \approx 3.5 \cdot 10^{-3}$, for convenience (2) can be reexpressed as

$$
E^{2}=1.67 \cdot 10^{-18} T_{e 0} v^{2}\left\{1-\left(\frac{v_{0}}{v}\right)^{6 / 5}\right\}
$$

where $E$ is in $\mathrm{V} / \mathrm{m}, T_{e r}$ in $\mathrm{K}, v_{0}$ and $v$ in $\sec ^{-1}$.

Thus, the electric field intensity can be estimated from partial reflection $v$ measurements using relations (2) and (3). The assumption of an undisturbed temperature profile $T_{e, t}$ readily defines a $v_{0}$ profile using the well-known relation (Gurevich, 1978)

$$
v_{0}=5.8 \cdot 10^{-11} N_{n} T_{e 0}^{5 / 6}
$$

where $N_{n}$ is the neutral particle number density at a particular altitude (in $\mathrm{cm}^{-3}$ ). Usually, we assume $v_{0} \approx \nu_{\mathrm{m}}$, although it is possible to determine $v_{0}$ from partial reflection data. However, our investigations show that the difference between model and experimental magnitudes of $\nu_{0}$ are usually of the order of partial reflection method errors, i.e. it does not exceed $30 \%$.

The histogram showing the distribution of $E$ derived from the histogram in Figure 1 using relation (3) is depicted in Figure 2. Here, $p_{E}=n_{E} / n_{l}$, where $n_{E}$ is the number of observations occurring in each $E$ bin, and $n$, is the same as for Figure 1. The data in Figure 2 exhibit an electric field intensity of $E>0.25 \mathrm{~V} / \mathrm{m}$ in about $70 \%$ cases at the lower edge of the ionosphere in our experiments. The $0-0.25 \mathrm{~V} / \mathrm{m}$ bin corresponds to both quiet geomagnetic conditions and small $E$ values of the order of random measurement errors in our estimates of $\nu$. The mean electric field magnitude $E$ as a function of altitude $z$ is as follows: $E(z=60 \mathrm{~km})=1.0 \pm 0.2 \mathrm{~V} / \mathrm{m}, E(z=63 \mathrm{~km})=0.5 \pm 0.1 \mathrm{~V} / \mathrm{m}, E(z=66 \mathrm{~km})=$ $0.3 \pm 0.1 \mathrm{~V} / \mathrm{m}$. The analysis of relations (2) and (3) shows that relative random measurement errors in $E$ are due to relative random measurement errors in $v$, and in our experiments do not exceed $30-50 \%$. 


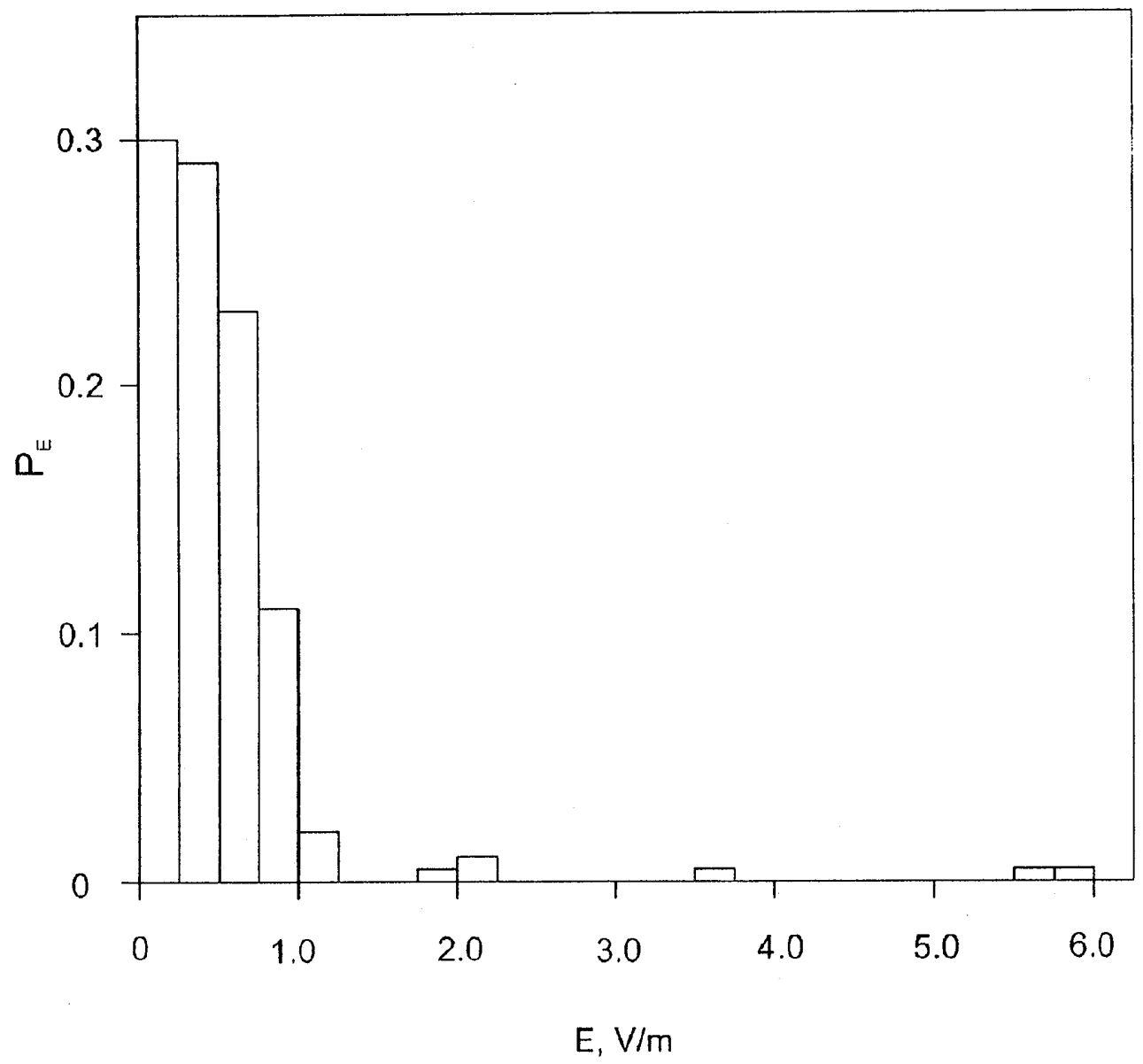

Fig. 2. Distribution of the electric field intensity values, $E$, under quiet conditions in an altitude range of $60-66 \mathrm{~km}$ in the daytime lower ionosphere at middle latitudes.

\section{Discussion}

The results obtained significantly improve the understanding of complicated physics of the disturbed ionospheric $\mathrm{D}$ region. The presence of significant electric fields at the lower edge of the ionosphere has provided evidence for an additional source of electron heating which should be taken into account while investigating a disturbed ionosphere and radio wave propagation in it. Besides, the results described above confirm the hypothesis that the anomalous changes in VLF signal phase observed on paths traversing regions of enhanced seismic activity during a few days before earthquakes and in the vicinity of nuclear power stations during accidents with the release of radioactive materials can be caused by changes in electric fields in the earth-electrosphere capacitor (Martynenko et al., 1996; Fuks et al., 1997). It should also be mentioned that the 
technique described above permits a real-time derivation of changes in electric field intensities from partial reflection measurements at the lower edge of the ionosphere.

\section{Summary}

The analysis of partial reflection signals from the lower edge of the ionosphere have yielded the distribution of effective electron collision frequency magnitudes derived with taking into account the kinetic effects. An assumption is made that this distribution is affected by strong atmospheric electric fields. A technique for derivation of these electric fields from effective electron collision frequency data is developed.

Acknowledgments. The authors have been supported by Science and Technology Center in Ukraine Grant No. 471.

\section{References}

Fuks, I. M., R. S. Shubova, and S. I. Martynenko, Lower ionosphere response to conductivity variations of the near-earth atmosphere, J. Atmos. Solar Terr. Phys., 59, 961-965, 1997.

Goldberg, R. A., Middle atmospheric electrodynamics: status and future, J. Atmos. Terr. Phys., 46, 1083-1101, 1984.

Gurevich, A. V., Nonlinear phenomena in the ionosphere, Springer-Verlag, New York, 1978.

Martynenko, S. I., Atmospheric electric field and disturbances of the lower ionosphere parameters, J. Atmos. Electricity, 19, 1999.

Martynenko, S. I., I. M. Fuks, and R. S. Shubova, Ionospheric electric-field influence on the parameters of VLF signals connected with nuclear accidents and earthquakes, $\mathrm{J}$. Atmos. Electricity, 16, 259-269, 1996.

Tyrnov, O. F., K. P. Garmash, A. M. Gokov, A. I. Gritchin, V. L. Dorohov, L. G. Kontzevaya, L. S. Kostrov, S. G. Leus, S. I. Martynenko, V. A. Misyura, V. A. Podnos, S. N. Pokhil'ko, V. T. Rozumenko, V. G. Somov, A. M. Tsymbal, L. F. Chernogor, and A. S. Shemet, The Radiophysical Observatory for remote sounding of the ionosphere, Turkish J. of Physics, 18, 1260-1265, 1994.

(Received November 28,1998; revised February 4,1999; accepted February 15,1999) 